\title{
Ketamine as Adjuvant for Acute Pain Management
}

\author{
Farnad Imani (iD ${ }^{1,{ }^{*}}$ and Giustino Varrassi (iD) ${ }^{2}$ \\ ${ }^{1}$ Pain Research Center, Iran University of Medical Sciences, Tehran, Iran \\ ${ }^{2}$ Paolo Procacci Foundation, Rome, Italy \\ "Corresponding author: Pain Research Center, Iran University of Medical Sciences, Tehran, Iran. Email: farnadimani@yahoo.com
}

Received 2019 December 14; Accepted 2019 December 16.

Keywords: Ketamine, Pain Management, Adjuvant

Peri-operative pain management is one of the important tasks of medical staff. The problems arising afterwards not only have a pathophysiological adverse effect on patients, but also detrimentally affect the health system, treatment costs, and delay in discharge, for which pain management should be planned prior to surgery, and the resulting stress (1-3).

Recent studies indicate a high incidence of persistent postoperative pain, and anesthesiologists should thus take proactive measures to control postoperative pain, particularly as some surgeries have more severe acute pain. Not only have a variety of drugs (such as opioids, NSAIDs, and adjuvants) been studied, but also several methods of administration (including oral, intravenous, epidural, inhalation, etc.) have been evaluated (4-6).

Because of having significant effect in pain management, opioids are the most commonly prescribed drugs, but considering their side effects, researchers have always been looking for alternative or adjunctive drugs and methods. Of adjuvants, paracetamol, ketamine, dexmedetomidine, gabapentin, pregabalin, lidocaine, amantandine, melatonin, and ketorolac, can be considered, in the meantime, for their role in post-operative pain management (79). As well as systemic administration of drugs, a variety of regional techniques including neuraxial and peripheral nerve blockade are of great attraction to anesthesiologists for pain management during and after surgery (10-12).

Opioids are the most prominent postoperative pain management drugs; however, the analgesic requirement may gradually grow up due to the possibility of hyperalgesia. Administration of sub-anesthetic doses of ketamine seems to be able to prevent this phenomenon, thereby preventing hypersensitivity and drug tolerance (13). In recent studies, special attention has been paid to ketamine. Ketamine is a noncompetitive inhibitor of NMDA receptors, having analgesic, anti-inflammatory, and anti- hyperalgesic effects, which has made it achieve a new position in postoperative pain management. Ketamine has not only been considered for acute postoperative pain management, but has also been used for peri-operative pain management, pre-emptive analgesia, multimodal analgesia, chronic pain, and controlling hemodynamic changes during intubation (14). In addition, as an adjuvant in regional anesthesia, such as peripheral nerve block, it has had significant effects (15). However, it is associated with some side effects, such as hemodynamic changes, hallucinations, and headaches, which may interfere with the postoperative recovery. Psychosomatic complications such as mood disorders and dissociative symptoms are among the most common, but transient; with problems associated with this drug disappearing usually in one hour. Central nervous system complications may also be present, but they are not common if administered as an adjuvant in patient controlled analgesia (PCA).

Ketamine administration has not been approved by the Food and Drug Administration (FDA) for neuraxial methods, and neurotoxicity has been observed in intrathecal continuous infusion of its racemic mixture, which, of course, appears to have occurred due to its preservative content, as no such cytotoxic effects have been observed in intrathecal administration of the preservative free type. Intranasal ketamine has a higher bioavailability than oral, and some studies have shown it to be effective in acute pain management following outpatient surgery procedures in children; psychosomatic effects have also been observed with this method, however (16).

In the studies available, the addition of ketamine to morphine has not always been associated with acute postoperative pain relief, and has had varied effects. In some studies it has delayed the time for the first postoperative analgesic request. On the contrary, low doses of ketamine during the operation has not affected morphine 
intake within the first 24 hours after cesarean section (17). Moreover, the addition of intravenous ketamine to epidural ropivacaine administration, after thoracotomy, has had significant effects on pain score reduction and opioid consumption, compared to the administration of epidural ropivacaine alone. On the other hand, a comparison of epidural ropivacaine infusion alone with subanesthetic $\mathrm{S}(+)$ ketamine infusion during thoracotomy, has shown that the latter has caused better postoperative analgesia, but has revealed no effect on abdominal hysterectomy and open colorectal surgery (18).

The different results observed with ketamine may be caused by various reasons, including the dosage of drug, the time of administration (intra- or post-operative period), the duration of postoperative administration, the method of administration of each drug (intravenous, epidural, or nasal), racemic ketamine or $\mathrm{S}(+)$ ketamine, duration of the postoperative evaluation, and single or combined administration with other drugs.

Ketamine is a suitable analgesic for use outside the operating room, especially in emergency conditions, as it maintains cardiovascular and respiratory stability in patients, while causing potent analgesia similar to opioids, but lacking a risk of respiratory depression (19). Nowadays, concomitant administration of ketamine with propofol or in a mixture called "Ketofol" is performed in some medical centers, used to induce analgesia and sedation in some procedures (procedural sedation).

Existing studies indicate that peri-operative ketamine administration has had favorable analgesic effects on postoperative acute pain, especially if it is combined with a multimodal approach, more considerable effects and fewer complications will be achieved. Furthermore, in many studies, ketamine has been suggested as a strong adjuvant in opioid analgesia, and appears to be capable of interfering with the peripheral and central transmission of the pain pathway. Ketamine has also been used in some chronic pain syndromes, such as post-herpetic neuralgia, neuropathic pain, fibromyalgia, spinal cord injuries, chronic regional pain syndrome, post-amputation pain, and cancer pains.

In the present issue of the journal, Zangouei and Zahraei (20) administered a low dose of ketamine following spinal anesthesia in cesarean section that reduced the incidence of postoperative headache. The incidence of headaches, of course, depends on a variety of factors, including patient age, needle size and shape, bevel orientation, inserting angle, multiple dural punctures, type of surgery, and physician experience, which should also be considered when the efficacy of administering a study drug to manage post-dural puncture headache (PDPH) is to be evaluated. The primary approach commonly used to treat or reduce the severity of PDPH are, initially, hydration, bed rest, and analgesics; the most effective treatment is a blood patch, though it is an invasive method with its own side effects.

Various medical therapies that have been used to prevent and reduce the incidence of post-dural puncture headache, including oral and intravenous caffeine, subcutaneous somatriptan, gabapentin, pregabalin, theophylline, hydrocortisone, and adrenocorticotropic hormone (ACTH), each with different therapeutic effects on PDPH. In a study, administration of aminophylline infusion during surgery has reduced the incidence of postdural puncture headache. In addition, placing the patient in the lateral decubitus position has reduced the incidence of post-dural puncture headache, and it can be taken into account during spinal anesthesia.

In the end, regardless of the beneficial effects of ketamine as an adjuvant in acute and chronic pain management, as well as post-dural puncture headache in some studies, the effects in other interventions should also be considered so that this theory about the effect of ketamine can be endorsed further.

\section{Footnotes}

Authors' Contribution: Study concept and design: Farnad Imani. Revision of the manuscript: Giustino Varrassi. Writing of the manuscript: Farnad Imani.

Conflict of Interests: The authors declared no conflict of interest.

Funding/Support: None.

\section{References}

1. Guimaraes-Pereira L, Valdoleiros I, Reis P, Abelha F. Evaluating persistent postoperative pain in one tertiary hospital: Incidence, quality of life, associated factors, and treatment. Anesth Pain Med. 2016;6(2). e36461. doi: 10.5812/aapm.36461. [PubMed: 27252908]. [PubMed Central: PMC4886451].

2. Khan TW, Imani F. The management of chronic pain; caught between a rock and a hard place: The Case for a renewed focus on provider, patient, and payer education. Anesth Pain Med. 2017;7(1). e40951. doi: 10.5812/aapm.40951. [PubMed: 28920037]. [PubMed Central: PMC5554427].

3. Malik KM, Beckerly R, Imani F. Musculoskeletal disorders a universal source of pain and disability misunderstood and mismanaged: A critical analysis based on the U.S. model of care. Anesth Pain Med. 2018;8(6). e85532. doi: 10.5812/aapm.85532. [PubMed: 30775292] [PubMed Central: PMC6348332].

4. Farzi F, Naderi Nabi B, Mirmansouri A, Fakoor F, Atrkar Roshan Z, Biazar G, et al. Postoperative pain after abdominal hysterectomy: A randomized, double-blind, controlled trial comparing the effects of tramadol and gabapentin as premedication. Anesth Pain Med. 2016;6(1). e32360. doi: 10.5812/aapm.32360. [PubMed: 27110531]. [PubMed Central: PMC4834422]. 
5. Rajappa GC, Vig S, Bevanaguddaiah Y, Anadaswamy TC. Efficacy of pregabalin as premedication for post-operative analgesia in vaginal hysterectomy.Anesth Pain Med.2016;6(3). e34591. doi:10.5812/aapm.34591. [PubMed: 27642577]. [PubMed Central: PMC5018136].

6. Yazdani J, Aghamohamadi D, Amani M, Mesgarzadeh AH, Maghbooli Asl D, Pourlak T. Effect of preoperative oral amantadine on acute and chronic postoperative pain after mandibular fracture surgery. Anesth Pain Med. 2016;6(3). e35900. doi: 10.5812/aapm.35900. [PubMed: 27642581]. [PubMed Central: PMC5018158].

7. Arhami Dolatabadi A, Memary E, Kariman H, Nasiri Gigloo K, Baratloo A. Intranasal desmopressin compared with intravenous ketorolac for pain management of patients with renal colic referring to the emergency department: A randomized clinical trial. Anesth Pain Med. 2017;7(2). e43595. doi: 10.5812/aapm.43595. [PubMed: 28824859]. [PubMed Central: PMC5556593].

8. Murthy Tk K, Kumar Pv V. Effect of perioperative intravenous lignocaine infusion on haemodynamic responses and postoperative analgesia in laparoscopic cholecystectomy surgeries. Anesth Pain Med 2018;8(2). e63490. doi: 10.5812/aapm.63490. [PubMed: 30009150]. [PubMed Central: PMC6035376].

9. Javaherforooshzadeh F, Amirpour I, Janatmakan F, Soltanzadeh M. Comparison of effects of melatonin and gabapentin on post operative anxiety and pain in lumbar spine surgery: A randomized clinical trial. Anesth Pain Med. 2018;8(3). e68763. doi: 10.5812/aapm.68763. [PubMed: 30214884]. [PubMed Central: PMC6119980].

10. Grant AE, Schwenk ES, Torjman MC, Hillesheim R, Chen AF. Postoperative analgesia in patients undergoing primary or revision knee arthroplasty with adductor canal block. Anesth Pain Med. 2017;7(3) e46695. doi: 10.5812/aapm.46695. [PubMed: 28824869]. [PubMed Central: PMC5559700].

11. Soliz JM, Lipski I, Hancher-Hodges S, Speer BB, Popat K. Subcostal transverse abdominis plane block for acute pain management: A review. Anesth Pain Med. 2017;7(5). e12923. doi: 10.5812/aapm.12923. [PubMed: 29696110]. [PubMed Central: PMC5903215].

12. Laulin JP, Maurette P, Corcuff JB, Rivat C, Chauvin M, Simonnet G. The role of ketamine in preventing fentanyl-induced hyperalgesia and subsequent acute morphine tolerance. Anesth Analg. 2002;94(5):12639. table of contents. doi: 10.1097/00000539-200205000-00040. [PubMed: 11973202].
13. Khajavi MR, Navardi M, Shariat Moharari R, Pourfakhr P, Khalili N, Etezadi $\mathrm{F}$, et al. Combined ketamine-tramadol subcutaneous wound infiltration for multimodal postoperative analgesia: A doubleblinded, randomized controlled trial after renal surgery. Anesth Pain Med. 2016;6(5). e37778. doi: 10.5812/aapm.37778. [PubMed: 27847695]. [PubMed Central: PMC5101596].

14. Zaman B, Hojjati Ashrafi S, Seyed Siamdoust S, Hassani V, Mohamad Taheri S, Noorizad S. The effect of ketamine and dexamethasone in combination with lidocaine on the onset and duration of axillary block in hand and forearm soft tissue surgery. Anesth Pain Med. 2017;7(5). e15570. doi: 10.5812/aapm.15570. [PubMed: 29696115]. [PubMed Central: PMC5903223].

15. Poonai N, Canton K, Ali S, Hendrikx S, Shah A, Miller M, et al. Intranasal ketamine for procedural sedation and analgesia in children: A systematic review. PLoS One. 2017;12(3). e0173253. doi: 10.1371/journal.pone.0173253. [PubMed: 28319161]. [PubMed Central: PMC5358746].

16. Reza FM, Zahra F, Esmaeel F, Hossein A. Preemptive analgesic effect of ketamine in patients undergoing elective cesarean section. Clin J Pain. 2010;26(3):223-6. doi: 10.1097|AJP.ob013e3181bff86d. [PubMed: 20173436].

17. Feltracco P, Barbieri S, Rizzi S, Ori C, Groppa F, De Rosa G, et al. Brief report: Perioperative analgesic efficacy and plasma concentrations of $\mathrm{S}+$-ketamine in continuous epidural infusion during thoracic surgery. Anesth Analg. 2013;116(6):1371-5. doi: 10.1213/ANE.0b013e31828cbafo. [PubMed: 23558843].

18. Grady MV, Mascha E, Sessler DI, Kurz A. The effect of perioperative intravenous lidocaine and ketamine on recovery after abdominal hysterectomy. Anesth Analg. 2012;115(5):1078-84. doi: 10.1213/ANE.0b013e3182662e01. [PubMed: 23011561].

19. Mahshidfar B, Mofidi M, Fattahi M, Farsi D, Hafezi Moghadam P, Abbasi S, et al. Acute Pain Management in Emergency Department, low dose ketamine versus morphine, a randomized clinical trial. Anesth Pain Med. 2017;7(6). e60561. doi: 10.5812/aapm.60561. [PubMed: 29696126]. [PubMed Central: PMC5903386].

20. Zangouei A, Zahraei SAH. Effect of low-dose intravenous ketamine on prevention of headache after spinal anesthesia in patients undergoing elective cesarean section: A double-blind clinical trial study. Anesth Pain Med. 2019;9(6). e97249. doi: 10.5812/aapm.97249. 